\title{
Assessing the Effects of Spatial Resolution on Regional Climate Model Simulated Summer Temperature and Precipitation in China: A Case Study
}

\author{
Xin-Min Zeng, ${ }^{1,2}$ Ming Wang, ${ }^{1,2}$ Yujian Zhang, ${ }^{3}$ Yang Wang, ${ }^{4}$ and Yiqun Zheng ${ }^{1,2}$ \\ ${ }^{1}$ College of Meteorology and Oceanography, PLA University of Science and Technology, Nanjing, Jiangsu, China \\ ${ }^{2}$ Key Laboratory for Mesoscale Severe Weather of Ministry of Education, Nanjing University, Nanjing, Jiangsu, China \\ ${ }^{3}$ Surveying, Mapping, and Meteorological Division, Unit 77200 of PLA, Kunming, China \\ ${ }^{4}$ Unit 93762 of PLA, Beijing, China
}

Correspondence should be addressed to Xin-Min Zeng; zen_xm@yahoo.com and Ming Wang; wangm_zlab@163.com

Received 5 December 2015; Accepted 18 January 2016

Academic Editor: Anthony R. Lupo

Copyright (C) 2016 Xin-Min Zeng et al. This is an open access article distributed under the Creative Commons Attribution License, which permits unrestricted use, distribution, and reproduction in any medium, provided the original work is properly cited.

\begin{abstract}
The regional climate model, RegCM3, is used to simulate the 2004 summer surface air temperature (SAT) and precipitation at different horizontal (i.e., 30, 60, and $90 \mathrm{~km}$ ) and vertical resolutions (i.e., 14, 18, and 23 layers). Results showed that increasing resolution evidently changes simulated SATs with regional characteristics. For example, simulated SATs are apparently better produced when horizontal resolution increases from 60 to $30 \mathrm{~km}$ under the 23 layers. Meanwhile, the SATs over the entire area are more sensitive to vertical resolution than horizontal resolution. The subareas present higher sensitivities than the total area, with larger horizontal resolution effects than those of vertical resolution. For precipitation, increasing resolution shows higher impact compared to SAT, with higher sensitivity induced by vertical resolution than by horizontal resolution, especially in rainy South China. The best SAT/precipitation can be produced only when the horizontal and vertical resolutions are reasonably configured. This indicates that different resolutions lead to different atmospheric thermodynamic states. Because of the dry climate and low soil heat capacity in Northern China, resolution changes easily modify surface energy fluxes, hence the SAT; due to the rainy and humid climate in South China, resolution changes likely strongly influence grid-scale structure of clouds and therefore precipitation.
\end{abstract}

\section{Introduction}

Using dynamical climate models to study climate change can help people understand climate variations. High-resolution regional climate models (RCMs) offer more advantages than general circulation models (GCMs) when used for regional climate simulations $[1,2]$. The higher resolutions of RCMs result in better representation of land surface heterogeneity and fine-scale forcing, for example, coastlines, complex topography, and land surface variations, which are all important for accurately simulating local and regional climate systems [3-7].

RCM research and applications include several works regarding the RegCM RCM model [8-13], in which the correlation of simulated temperatures with observations was higher than that of simulated precipitation $[14,15]$.
In horizontal resolution studies, Giorgi and Marinucci [8] showed that precipitation, clouds, land surface energy flux, and precipitation intensity distribution are more sensitive to horizontal resolution than topographic forcing. In addition, these authors noted that the sensitivity of physical schemes to resolution significantly alters the function of the topographic forcing. Leung and Qian [16] found that higher horizontal resolutions can improve the results of precipitation simulations along coastal slopes and in basin areas but could also overestimate precipitation in some areas. When conducting numerical simulations of East Asian precipitation, Gao et al. [17] found that the simulated results of smooth terrain at high resolution are better than those of actual terrain at low resolution and suggested that the role of terrain is equivalent to the role of spatial resolution. Kendon et al. [18] indicated that simulated precipitation at higher resolution 
is much more realistic and tends to increase the intensity of heavy rainfall with improved duration and areal extent. Chan et al. [19] simulated extreme precipitation and obtained high-resolution RCM results that were realistic for June through August. Hui et al. [20] showed that high-resolution simulations are more proficient at modeling mean climate and correlation coefficients. However, although most of these studies of the effects of resolution used RegCM, they did not consider the effects of vertical resolution on the simulated climate.

Few studies have considered the effects of vertical resolution in RCM simulations. For example, Ruti et al. [21] suggested that higher vertical resolution could improve precipitation simulations by affecting the cloud structure. Tang et al. [22] used MM5 to study extreme climatic events and showed that increasing the horizontal and vertical resolution can improve the simulation of heavy rainfall and SAT. Druyan et al. [23] noted that increasing the vertical resolution could strengthen atmospheric circulation and vertical wind shear when the horizontal resolution is fixed. Delworth et al. [24] showed that using a high resolution GFDL CM2.5 greatly improved regional precipitation features. Most of these studies stressed the significance of using appropriate vertical resolutions for precipitation simulations using different RCMs.

The above studies on the effects of resolution in RCM simulations have two features. (1) Most studies focused on the impacts of resolution on simulated precipitation, and few studies focused on both SAT and precipitation. SAT and precipitation are the two most important surface climate characteristics. Because of the influences of water and heat balances, some inherent variations exist between these surface climate characteristics. Previous studies have shown that decadal climate covariabilities exist between the summer SAT and soil moisture (note that precipitation can be influenced by soil moisture) under the influence of global warming in China [25]. However, Zeng and Xi [26] carried out a dynamic forecast of regional climate and found that the correlation between precipitation and SAT at the monthly scale is not high. Therefore, studies of the impacts of resolution on simulated SATs and precipitation are also meaningful at the seasonal scale. (2) Most studies have focused on the effects of horizontal resolution on regional climate simulations. When discussing the influence of vertical resolution on RCM capabilities, the effects of two or more vertical resolutions are seldom discussed. Therefore, it is difficult to ascertain details regarding the sensitivity of simulation results to vertical resolution.

Therefore, to study the effects of the different horizontal and vertical resolutions of RegCM3 on SAT and precipitation and the correlations between them over China, we simulated the 2004 summer climate using three different horizontal and vertical resolutions. Note that we used version 3 of RegCM, while the newest version is RegCM4.5; the findings in this paper are suggested to be version-independent. Hence, we have arranged the paper as follows. First, the RegCM3 model is briefly described and the experiments are designed in Section 2. Then, the effects of resolution on SAT, precipitation, and the correlation between temperature and
TABLE 1: Designed experimental schemes based on horizontal resolutions.

\begin{tabular}{lcc}
\hline Scheme & Horizontal resolution & Simulation area (buffer) grid \\
\hline H3 & $30 \mathrm{~km}$ & $171 \times 171(24 \times 24)$ \\
H6 & $60 \mathrm{~km}$ & $85 \times 85(12 \times 12)$ \\
H9 & $90 \mathrm{~km}$ & $57 \times 57(8 \times 8)$ \\
\hline
\end{tabular}

precipitation are discussed in Section 3. Finally, a summary and discussion are given in Section 4.

\section{Methods}

2.1. Model and Designed Experiments. The regional climate model (RegCM3) used in this work was developed by the Abdus Salam International Centre for Theoretical Physics (ICTP) [27]. The RegCM3 model is a representative regional climate model that is used widely for research and in other applications $[28,29]$. Based on the hydrostatic version of the fifth-generation Pennsylvania State University-National Center for Atmospheric Research Mesoscale Model (PSUNCAR_MM5), the dynamical core of its sigma-vertical coordinate model is hydrostatic and compressible [30].

Due to many factors, such as mathematical calculation errors in numerical models and the applicability of physical parameterization schemes that are relevant to spatial extent and scale, different spatial resolutions are expected to result in different simulation results. To test differences in resolution, we used the same physical parameterization schemes for each experiment (Tables 1 and 2). The physical parameterization schemes adopted in this study include the planetary boundary layer scheme of Holtslag et al. [31], the Subgrid Explicit Moisture Scheme (SUBEX) [32], and the Grell cumulus parameterization scheme [33]. In addition, radiation was represented by version 3 of the Community Climate Model (CCM3) that was parameterized by Kiehl et al. [34]. In each experiment, the simulation domain was centered at $\left(102^{\circ} \mathrm{E}, 35^{\circ} \mathrm{N}\right)$ and the simulation area covered mainland China and adjacent sea areas (Figure 1). The three horizontal resolutions adopted here have the following three features that favor the comparison of results. (1) The three horizontal resolutions are typical spatial resolutions used for current regional climate simulations and can represent three types of horizontal resolution (high, medium, and coarse horizontal resolutions). (2) The horizontal resolutions have a linear incremental relationship; thus, it is possible to discuss the consistency of simulation results for different horizontal resolutions at a given vertical resolution and study the coordination of the vertical and horizontal resolutions. (3) In all the experiments, the simulation domains, buffer zones, and areas of analysis are the same. This type of experimental design enhances the comparability of the different simulation results compared with previous research using RCMs.

According to nearly 30 years of variations in summer SAT and precipitation (Figure 2), a number of years (e.g., 1988 and 2004) were climatologically typical. Considering the computational cost, as an example, all simulations in this 
TABLE 2: Designed experimental schemes based on different vertical layers with different sigma $(\sigma)$ values.

\begin{tabular}{|c|c|c|c|c|}
\hline Scheme & Layer & $\sigma>0.8$ & $0.8 \leq \sigma \leq 0.2$ & $\sigma<0.2$ \\
\hline V14 & 14 layers & $\begin{array}{c}1,0.99,0.97,0.93 \\
0.86\end{array}$ & $0.77,0.67,0.56,0.46,0.35,0.25$ & $0.17,0.1,0.04$ \\
\hline V18 & 18 layers & $\begin{array}{c}1,0.99,0.98,0.96 \\
0.93,0.89,0.84 \\
\end{array}$ & $\begin{array}{c}0.78,0.71,0.63,0.55,0.47,0.39 \\
0.31,0.23\end{array}$ & $0.16,0.1,0.05$, \\
\hline V23 & 23 layers & $\begin{array}{c}1,0.99,0.98,0.96 \\
0.93,0.89,0.85\end{array}$ & $\begin{array}{c}0.8,0.75,0.7,0.65,0.6,0.55,0.5 \\
0.45,0.4,0.35,0.3,0.25,0.2\end{array}$ & $0.15,0.1,0.05$ \\
\hline
\end{tabular}

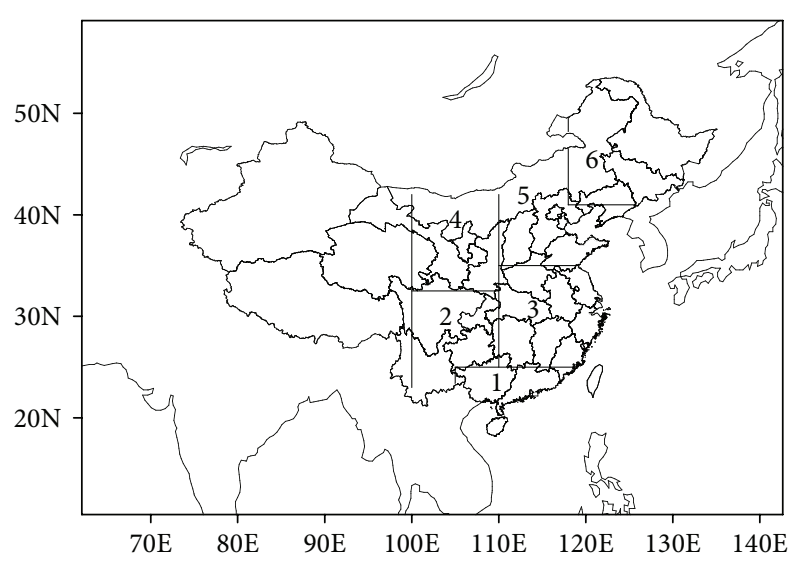

Figure 1: Model domain and distribution of the studied subareas, where subareas 1-6 correspond to South China, Southwest China, East China, Northwest China, North China, and Northeast China, respectively.

paper were carried out from 1 May 2004 to 1 September 2004. The NCEP $2.5^{\circ} \times 2.5^{\circ}$ reanalysis data were used as the initial and boundary conditions for all simulations. The first month (i.e., May) of integrations was used for model spin-up, and the sensitivity of the summer (June, July, and August; JJA) climate was analyzed for resolution differences. Because RCM ensemble results using different initial-value perturbations have only shown small spreads for variables [35], we only carried out one deterministic simulation for each horizontal/vertical resolution. To study the capacities of RCM simulations in different regions of China, we roughly divided mainland China into six subareas (i.e., South China, Southwest China, East China, Northwest China, North China, and Northeast China; Figure 1). The total area is the land surface of the entire simulation domain except the buffer zone, and its size exceeds the sum of the six subareas.

2.2. The Data. We used NCEP/NCAR $2.5^{\circ} \times 2.5^{\circ}$ reanalysis data [36] to model the initial and boundary conditions. To validate the model results, the monthly precipitation and SAT data from Willmott et al. [37] were used. To fit the selected resolutions used for different simulations, the United States Geological Survey (USGS) $30-\mathrm{sec}(\sim 1 \mathrm{~km})$ elevation data were used after averaging the data for each grid cell.

\section{Results and Discussion}

In this paper, only the average seasonal results are discussed. To compare the simulated results and observations that were obtained from the Climate Research Center in the Department of Geography at the University of Delaware and to study the differences between various experiments, we define the seasonal mean model bias (BIAS), root mean square error (RMSE), correlation coefficient (CORR), and standard deviation (STD; $\mathrm{STD}_{M}$ and $\mathrm{STD}_{\mathrm{O}}$ for simulations and observations, resp.) as follows [35]:

$$
\begin{aligned}
\mathrm{BIAS} & =\bar{M}-\bar{O}, \\
\mathrm{RMSE} & =\sqrt{\frac{1}{N} \sum_{i=1}^{N}\left(M_{i}-O_{i}\right)^{2}}, \\
\mathrm{CORR} & =\frac{\sum_{i=1}^{N}\left(M_{i}-\bar{M}\right)\left(O_{i}-\bar{O}\right)}{\sqrt{\sum_{i=1}^{N}\left(M_{i}-\bar{M}\right)^{2}} \sqrt{\sum_{i=1}^{N}\left(O_{i}-\bar{O}\right)^{2}}}, \\
\operatorname{STD}_{M} & =\sqrt{\frac{\sum_{i=1}^{N}\left(M_{i}-\bar{M}\right)^{2}}{N}}, \\
\operatorname{STD}_{O} & =\sqrt{\frac{\sum_{i=1}^{N}\left(O_{i}-\bar{O}\right)^{2}}{N}},
\end{aligned}
$$

where $N$ is the total number of stations within a region and $M$ and $O$ represent the observed and simulated values of a quality, respectively. Therefore, BIAS reflects the systematic bias of the regional model and RMSE represents the mean value of the deviation of a quantity from the observations in a region. In general, greater absolute BIAS values correspond with greater RMSE values, and higher spatial correlation coefficients (CORRs) indicate closer agreement between the simulated and observed values. When the standard deviations of the simulations $\left(\mathrm{STD}_{M}\right)$ are closer to the standard deviations of the observations $\left(\mathrm{STD}_{\mathrm{O}}\right)$, the spatial variability is simulated more accurately.

3.1. Effects on Simulated SAT. Figure 3 shows the BIAS and RMSE values of the SAT for the simulated subareas during the summer. Figure 3(a) shows that the simulated SATs are negatively biased for each subarea except Northwest China in 


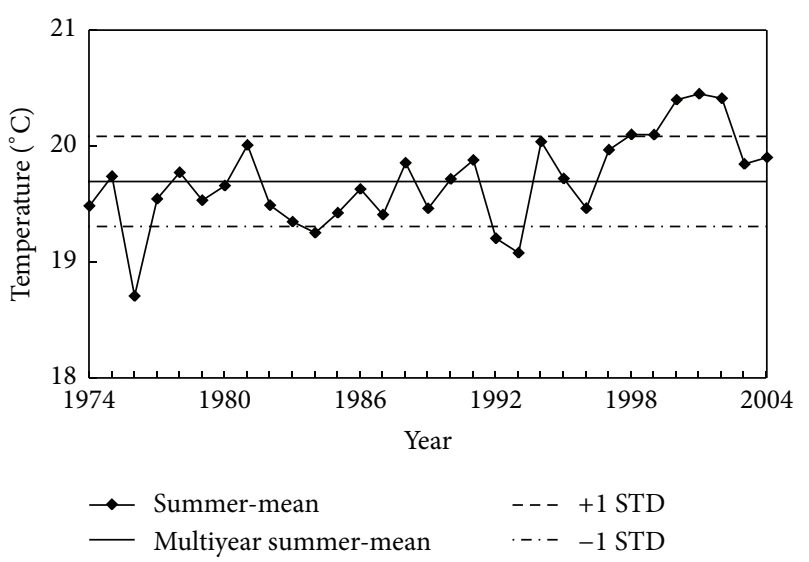

(a) SAT change

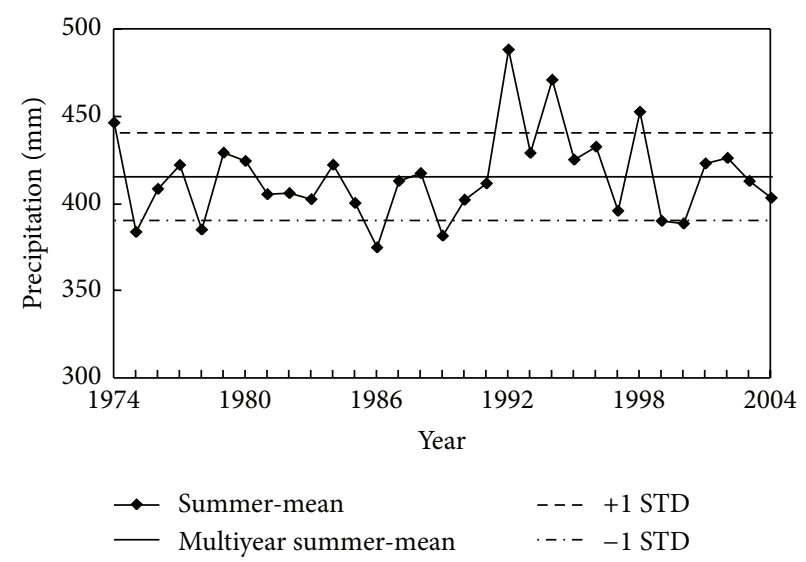

(b) Precipitation change

FIGURE 2: Variations of summer SAT and precipitation over the past 30 years in mainland China.

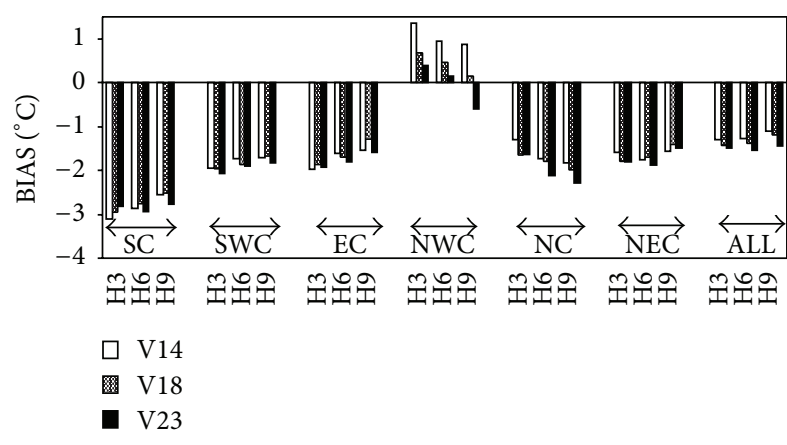

(a) SAT bias

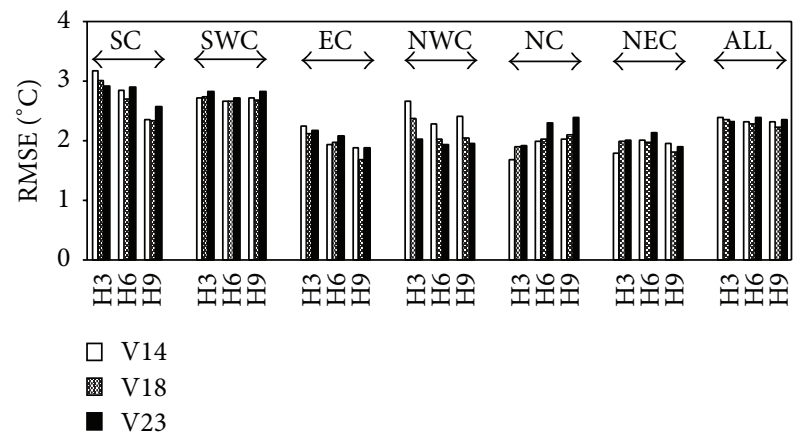

(b) SAT root mean square errors

Figure 3: BIAS (a) and RMSE (b) of summer-mean SAT for the studied subareas (South China (SC), Southwest China (SWC), East China (EC), Northwest China (NWC), North China (NC), Northeast China (NEC), and total area (ALL)).

all experiments. This result is consistent with the conclusions of Lian and Shu [38], who found that the RegCM3 summer SATs were lower than the actual temperatures observed in China. The effects of horizontal resolution on SAT at different vertical resolutions will be examined in this section.

\subsubsection{Horizontal Resolution Effects}

(a) Preliminary Results of Scheme V23. By comparing the distributions of observations with simulated summer SATs using different horizontal resolutions in China under Scheme V23, it appears that the temperature distributions are simulated well at all three horizontal resolutions. The SATs in East China tend to decrease with increasing latitude in areas of relatively flat land (Figure 4). The simulated cold spot located on the Tibetan Plateau is caused by the high topographic elevation, and the simulated warm spots located at $\left(28^{\circ}-\right.$ $\left.32^{\circ} \mathrm{N}, 105^{\circ}-110^{\circ} \mathrm{E}\right)$ and $\left(42^{\circ}-50^{\circ} \mathrm{N}, 80^{\circ}-90^{\circ} \mathrm{E}\right)$ are roughly consistent with the observations. Comparing the simulated temperature distributions at the three horizontal resolutions demonstrates that the distributions are consistent; however, simulations with higher horizontal resolutions display greater temperature detail at smaller scales. This increased detail can be justified theoretically. Higher horizontal resolutions correspond with finer computational grids depicting the topography and coastline of the simulated region; therefore, details at smaller scales can be forced by using finer land representations. In addition, these details are generally closer to the observations (e.g., the relatively high SAT of Sichuan Basin $\left.\left(28^{\circ}-32^{\circ} \mathrm{N}, 105^{\circ}-110^{\circ} \mathrm{E}\right)\right)$, and higher horizontal resolution results in temperature centers that are closer to the observed values.

Figure 3(a) shows that the difference in BIAS for the total area from the three experiments is small, with BIAS values of $-1.45,-1.5$, and $-1.53^{\circ} \mathrm{C}$ for $\mathrm{H} 6, \mathrm{H} 3$, and $\mathrm{H} 9$, respectively. In the subareas, the absolute BIAS values of North China decrease as the horizontal resolution increases. In Northeast China, the H9 SAT is the best, and the H6 absolute bias value is the largest. In Northwest China, the sign of the BIAS changes from positive to negative as the horizontal resolution increases. Hence, large differences can be observed in the summer temperature simulations for different subareas.

Meanwhile, the RMSEs for the total area (Figure 3(b)) in the simulated results obtained from the $\mathrm{H} 3, \mathrm{H} 6$, and $\mathrm{H} 9$ experiments are $2.34,2.37$, and $2.31^{\circ} \mathrm{C}$, respectively. When considering the correlation coefficients (Table 3 ), the H6 


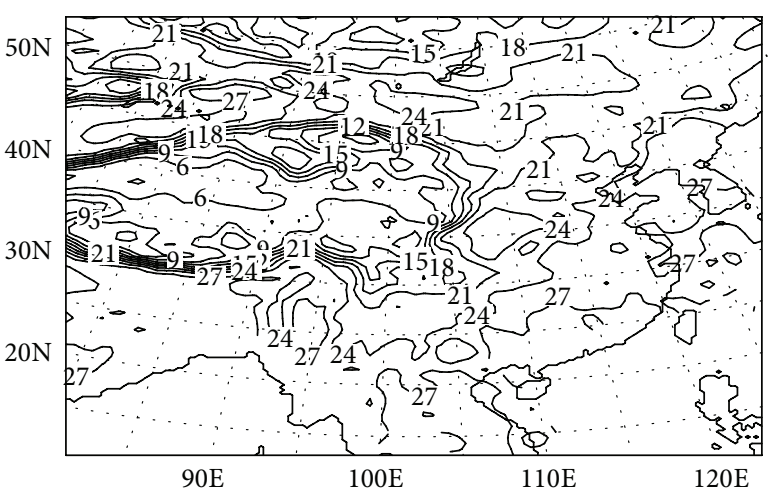

(a) Observation

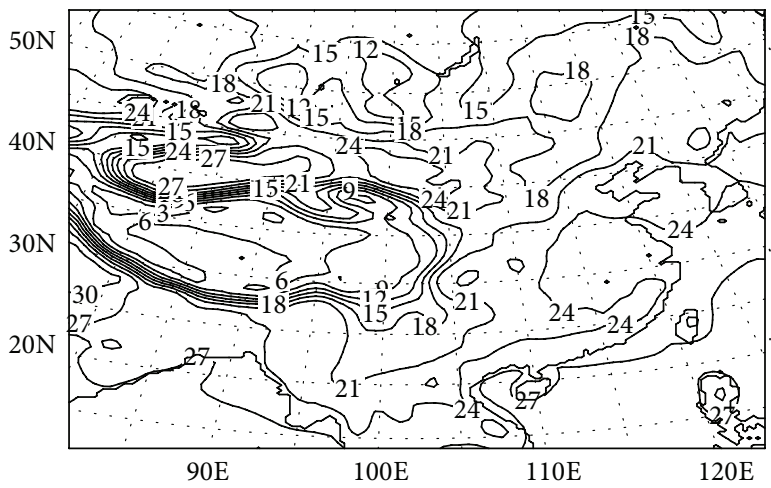

(c) $\mathrm{V} 23 \mathrm{H} 6$

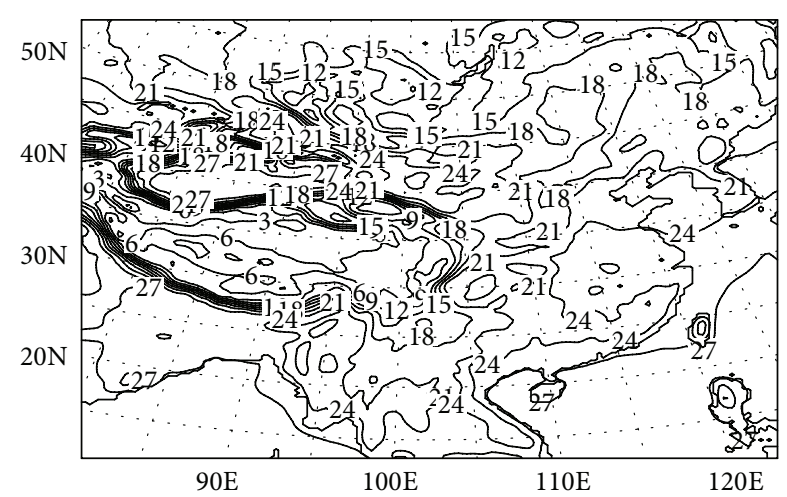

(b) $\mathrm{V} 23 \mathrm{H} 3$

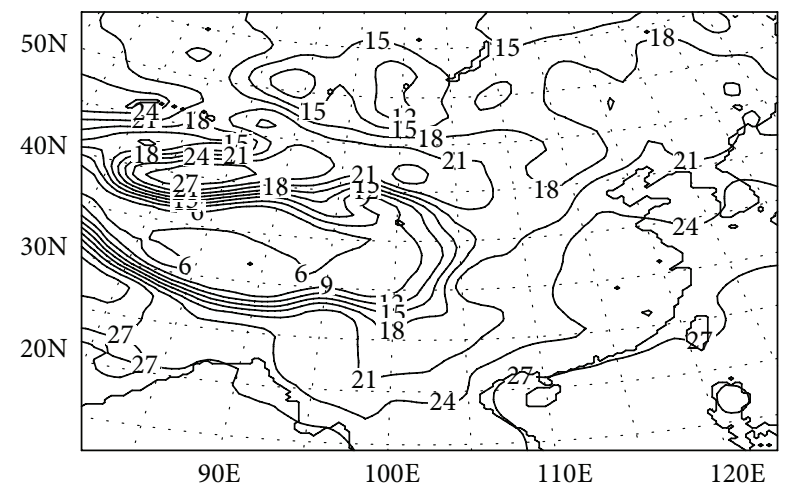

(d) $\mathrm{V} 23 \mathrm{H} 9$

FIGURE 4: Summer-mean distribution of SAT from observations and simulations using Scheme V23 for (a) observations, (b) H3, (c) H6, and (d) $\mathrm{H} 9$ in ${ }^{\circ} \mathrm{C}$.

TABLE 3: Spatial correlation coefficients of SAT for the V23 and H3 Schemes at different resolutions for the subareas.

\begin{tabular}{lccccccc}
\hline Test & SC & SWC & EC & NWC & NC & NEC & ALL \\
\hline V23H9 & 0.781 & 0.911 & 0.669 & 0.926 & 0.896 & 0.772 & 0.964 \\
V23H6 & 0.813 & 0.923 & 0.719 & 0.938 & 0.917 & 0.859 & 0.966 \\
V23H3 & 0.822 & 0.931 & 0.783 & 0.938 & 0.927 & 0.912 & 0.968 \\
V18H3 & 0.829 & 0.932 & 0.772 & 0.923 & 0.928 & 0.913 & 0.964 \\
V14H3 & 0.812 & 0.931 & 0.745 & 0.927 & 0.921 & 0.914 & 0.959 \\
\hline
\end{tabular}

temperature shows almost no improvement relative to the $\mathrm{H} 9$ temperature. However, $\mathrm{H} 3$ presents better temperatures than H6. In addition, the differences in the standard deviations of $\mathrm{H} 3, \mathrm{H} 6$, and $\mathrm{H} 9$ are very small, with all $\mathrm{STD}_{M}$ values similar to the $\mathrm{STD}_{O}$ values (Figure 5(a)). This result indicates that Scheme V23 successfully simulates the spatial variability of the temperature.

For each subarea, the CORR values (Table 3) show a marked increase with increasing horizontal resolution. Meanwhile, the RMSE values in North China decrease with increasing horizontal resolution, and the opposite results occur in East and South China. This result suggests an inconsistency when CORR is used to assess the simulation results in constructions with BIAS and RMSE. The H6 RMSE is smallest in Southwest and Northwest China and largest in Northeast China when compared with those of the other subareas.

For Scheme V23, the changes in SAT with horizontal resolution can be substantial in different areas, while the changes in the total area are slight (Figure 7).

(b) Comparison of Schemes V14 and V18 with Scheme V23. Figure 3(a) shows the V18 BIAS values of H9, H6, and H3 in the total area (i.e., $-1.16,-1.35$, and $-1.42^{\circ} \mathrm{C}$, resp.). In the subareas, the corresponding absolute BIAS values (i.e., 1.96, 1.77 , and $1.62^{\circ} \mathrm{C}$, resp.) in North China consistently decrease, and the values in the other subareas generally increase. For the RMSE, the values of the total area increase with increasing horizontal resolution. However, the degree of this increase is not substantial. For the subareas, the RMSE values are very sensitive to horizontal resolution, for example, 2.09, 2.00, and $1.89^{\circ} \mathrm{C}$ in North China and $2.31,2.68$, and $3.00^{\circ} \mathrm{C}$ in South China for $\mathrm{H} 9, \mathrm{H} 6$, and $\mathrm{H} 3$, respectively. These differences clearly indicate the high sensitivity of simulated SAT to horizontal resolution when considering a summer SAT increase of $0.15^{\circ} \mathrm{C}$ per decade in China during the last 50 years [39].

For Scheme V14, the H9, H6, and H3 BIAS values in the total area are $-1.10,-1.28$, and $-1.31^{\circ} \mathrm{C}$, respectively. The effects of horizontal resolution are greater in the subareas 


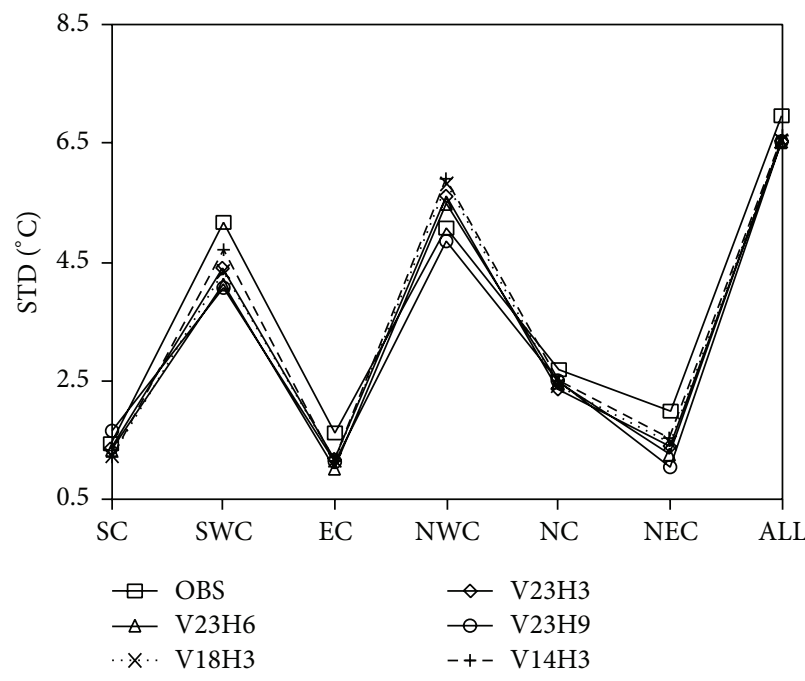

(a) SAT standard deviation

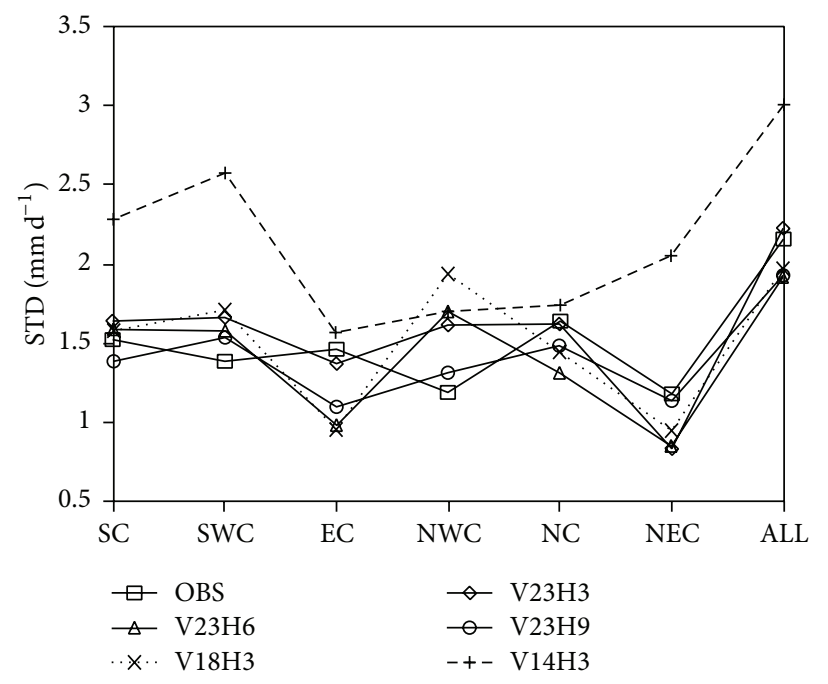

(b) Precipitation standard deviation

FIgURE 5: The standard deviation of SAT and precipitation at different resolutions in the studied subareas.

than in the total area, for example; the H9-H3 difference amounts to $0.5^{\circ} \mathrm{C}$ in South China. Moreover, when the horizontal resolution increases for Scheme V14, the RMSE only continuously decreases over North China. Meanwhile, for the total area, the H3 RMSE is larger than the RMSEs of $\mathrm{H} 6$ and $\mathrm{H} 9$.

As shown in Figure 3(a), the V23 SAT does not systematically move closer to the observed values as the horizontal resolution increases; for example, $\mathrm{H} 9, \mathrm{H} 6$, and $\mathrm{H} 3$ present the medium, largest, and lowest (the lowest, medium, and largest) BIAS amplitudes for the total area (East China). For Schemes V14 and V18, the simulated SATs deviate even further from the observations of the total area as the horizontal resolution increases. For Scheme V23, the largest BIAS difference (i.e., $0.08^{\circ} \mathrm{C}$ ) is found between $\mathrm{H} 6$ and $\mathrm{H} 9$ in the total area, and the largest RMSE difference (i.e., $0.05^{\circ} \mathrm{C}$ ) exists between $\mathrm{H} 3$ and H6. However, for Scheme V18, the largest BIAS (RMSE) difference exists between $\mathrm{H} 3$ and $\mathrm{H} 9$, with a value of $0.26^{\circ} \mathrm{C}\left(0.12^{\circ} \mathrm{C}\right)$. Similar results are found for Scheme V14. These differences suggest that the effects of increasing horizontal resolution are nonlinear at different vertical resolutions. Moreover, at a given vertical resolution, the sensitivity of the simulated SATs to horizontal resolution is different; for example, the sensitivity is the highest in Scheme V18 and the lowest in Scheme V23. In all subareas except for South and Northeast China, the influences of increasing horizontal resolution on simulated SAT are linear. Meanwhile, the simulated SATs are more sensitive to vertical resolution (at a given horizontal resolution) than horizontal resolution (at a given vertical resolution); for example, for the total area (Northwest China), the largest BIAS difference among the simulations induced by the horizontal resolution is $0.29^{\circ} \mathrm{C}\left(0.45^{\circ} \mathrm{C}\right)$ and the largest BIAS difference induced by the vertical resolution is $0.40^{\circ} \mathrm{C}\left(1.26^{\circ} \mathrm{C}\right)$.

\subsubsection{Vertical Resolution Effects}

(a) Preliminary Results of Resolution H3. Overall, the simulated H3 SATs are lower than the observations (Figure 3(a)). The amplitudes of the negative BIAS values for the total area consistently increase $\left(-1.31,-1.42\right.$, and $-1.50^{\circ} \mathrm{C}$ for V14, V18, and V23, resp.) with increasing vertical resolution, potentially because the larger positive BIAS in Northwest China is offset by the negative bias values in the other subareas. In the subareas, the V14 SATs decrease significantly in North and Northeast China, and the variations in the simulation results in Northwest and South China contrast those of the total area with increasing vertical resolution. The RMSE values of the total area decrease continuously and slightly $(2.37,2.33$, $2.31^{\circ} \mathrm{C}$ ) with increasing vertical resolution. In all subareas except South and Northwest China, where the SAT results are similar to those of the total area, and East China, where the V18 and V14 values are the smallest and the largest, respectively, the RMSE values of the other subareas increase with increasing vertical resolution.

The spatial correlation coefficients for the total area and the subareas are slightly different in the SAT distributions among V14, V18, and V23. In addition, these distributions are very similar to the observed SATs (Table 3 ). This result indicates that the influence of increasing vertical resolution on the SAT distribution is negligible. In addition, the $\mathrm{STD}_{M}$ values (Figure 5(a)) are similar to the observations in the different subareas. Overall, higher vertical resolution may not improve the simulation results at a resolution of $30 \mathrm{~km}$.

(b) Comparison of the H6 and H9 Resolutions with the H3 Resolution. The H6 SATs are similar to those of H3, and the absolute BIAS values for the total area increase with increasing vertical resolution. In the subareas, except in Northeast and South China, where the V18 absolute BIAS 


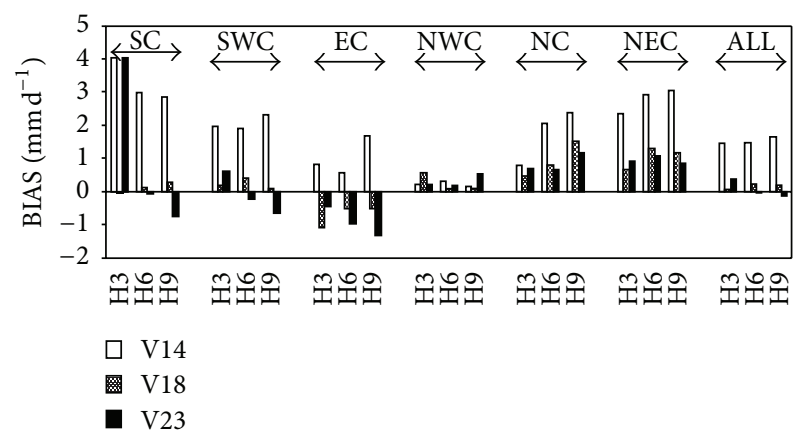

(a) Precipitation bias

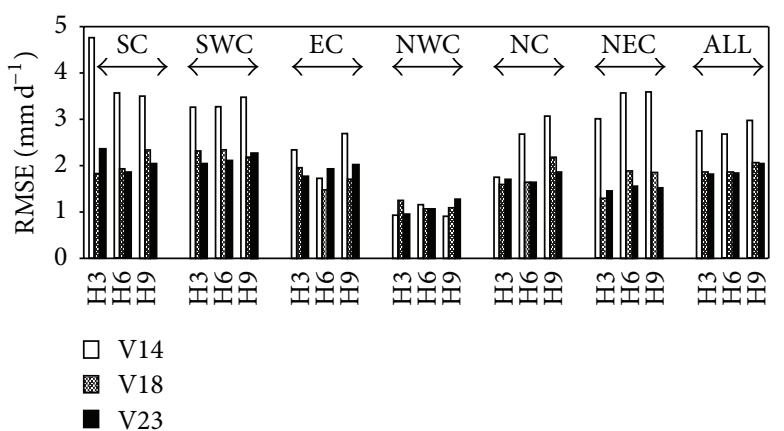

(b) Precipitation root mean square errors

FIGURE 6: Bias (a) and RMSE (b) of summer-mean precipitation for the studied areas.

value is the smallest, and in Northwest China, where the BIAS values decrease with increasing vertical resolution, SAT results that are similar to those of the total area are presented. When considering the RMSE, the V18 value is the smallest for the total area, and the differences between the three experiments are very small. For each subarea, the RMSE change is different for different subareas and varies with vertical resolution; for example, the RMSE values for East and North China increase with increasing vertical resolution and those for Northwest China decrease with increasing vertical resolution. Scheme H9 shows SAT results that are very similar to the $\mathrm{H} 6$ values for the total area and all the subareas with changing vertical resolution, except for the V18 absolute BIAS and RMSE values, which are smaller.

The SATs for the $\mathrm{H} 6, \mathrm{H} 9$, and $\mathrm{H} 3$ resolutions can differ greatly. For the total area, V23 produces slightly better SATs than V14 and V18 at the H3 resolution. For resolutions $\mathrm{H} 6$ and $\mathrm{H} 9, \mathrm{~V} 23$ provides results that deviate more from the observations than V14 and V18. In addition, the largest differences in the BIAS values for the V14 and V18 simulations are 0.25 and $0.35^{\circ} \mathrm{C}$, respectively, which correspond to the largest RMSE differences of 0.1 and $0.13^{\circ} \mathrm{C}$, respectively. The SAT sensitivity to vertical resolution is higher than its sensitivity to horizontal resolution. In the subareas, the sensitivities of the simulated SATs to vertical resolution are weak for different horizontal resolutions. Simultaneously, the simulations of SAT are more sensitive to horizontal resolution than vertical resolution.

3.2. Effects on Simulated Precipitation. Figure 6 shows the BIAS and RMSE values of the simulated summer precipitation for the studied areas. The BIAS values (Figure 6(a)) indicate that the simulated precipitation is higher than the observed precipitation over most of the subareas and that all the values in Northern China (Northwest China, North China, and Northeast China) are higher than the observations. In addition, the changes in BIAS and RMSE induced by the resolution change are all significant.

\subsubsection{Horizontal Resolution Effects}

(a) Preliminary Results of Scheme V23. Compared to the observations, the distributions of the simulated precipitation at the three horizontal resolutions and V23 (23 layers) are well reproduced; for example, precipitation appears to decrease from the southeast coast to the northwest inland region. In addition, the model generally simulates a large amount of false precipitation on the Tibetan Plateau due to megarelief. The effects of horizontal resolution on simulated precipitation have features similar to the effects on simulated SATs; that is, at higher horizontal resolutions, smaller-scale details can be identified and the precipitation centers are better produced.

Precipitation BIAS values (Figure 6(a)) indicate that the predicted $\mathrm{H} 6$ and $\mathrm{H} 9$ precipitation values are lower than the observations for the total area. However, $\mathrm{H} 3$ produces precipitation values that are greater than the observations, and the precipitation predicted by $\mathrm{H} 6$ is closest to the observed values. In the subareas, the $\mathrm{H} 3, \mathrm{H} 6$, and $\mathrm{H} 9$ precipitation values in Northern China are all greater than the observations. In South and Southwest China, all experiments are consistent with those of the total area; that is, the simulated amount of precipitation increases from $\mathrm{H} 9$ to $\mathrm{H} 3$. However, East China consistently presents simulated precipitation amounts that are lower than the observed amounts of precipitation at the three resolutions. These results are consistent with those of Zhang et al. [40], whose simulations indicated that RegCM3 tends to produce less (more) precipitation than the observed precipitation in Southern (Northern) China, indicating that the simulation results are related to the amount of precipitation [41]. In the subareas, the absolute BIAS values in East China decrease as the horizontal resolution increases. In Northeast China, H9 performs the best, and H6 BIAS is the largest. In Southwest China, the largest difference is $20 \%$, which shows the large sensitivity of regional precipitation to changes in horizontal resolution.

The RMSE values for the total area decrease as the horizontal resolution increases (Figure 6(b)). However, the decrease of $\mathrm{H} 3$ is not apparent compared with that of $\mathrm{H} 6$. The total area experiences a CORR increase with increasing horizontal resolution (Table 4). These results clearly indicate that $\mathrm{H} 6$ is an improvement over $\mathrm{H} 9$, while $\mathrm{H} 3$ does not perform superior to H6. In addition, the standard deviation values (Figure 5(b)) show that the simulations with higher horizontal resolutions for the total area better represent the spatial variability. However, some discrepancies exist in the studied subareas. For example, for H6 in South China, the 


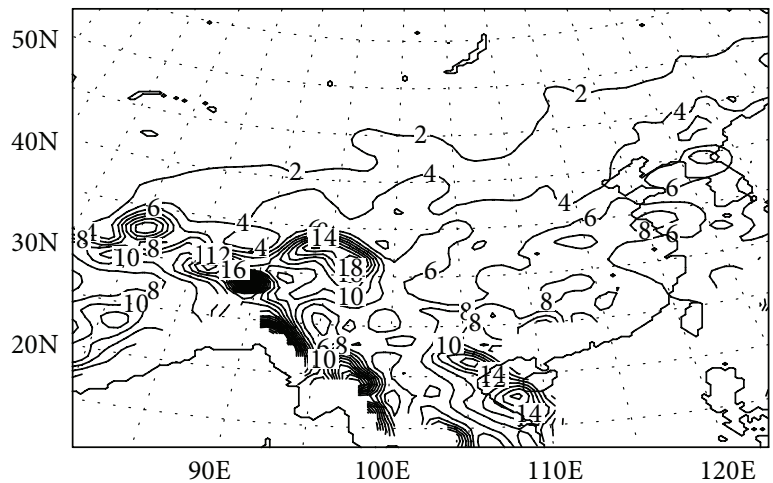

(a) Observation

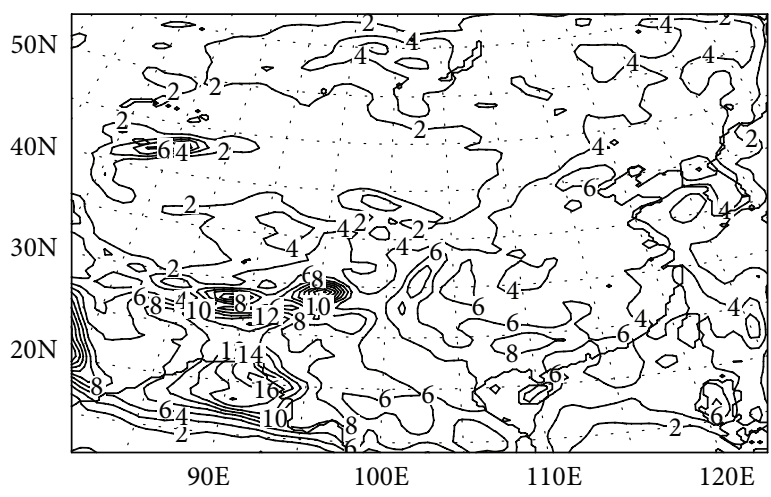

(c) $\mathrm{V} 23 \mathrm{H} 6$

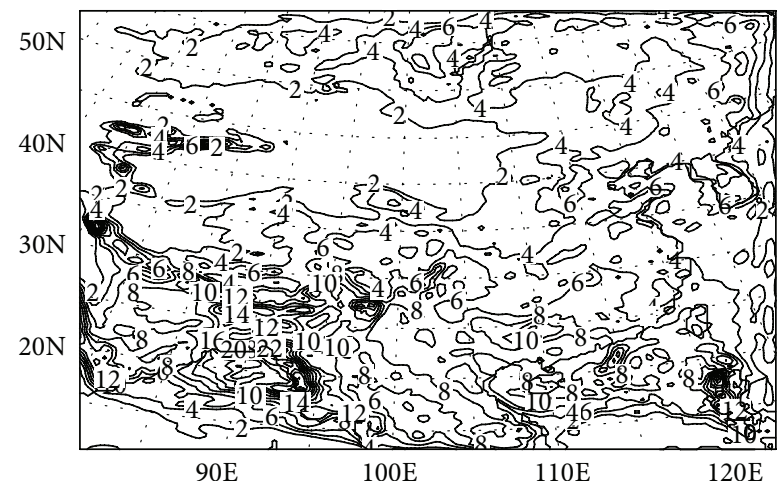

(b) $\mathrm{V} 23 \mathrm{H} 3$

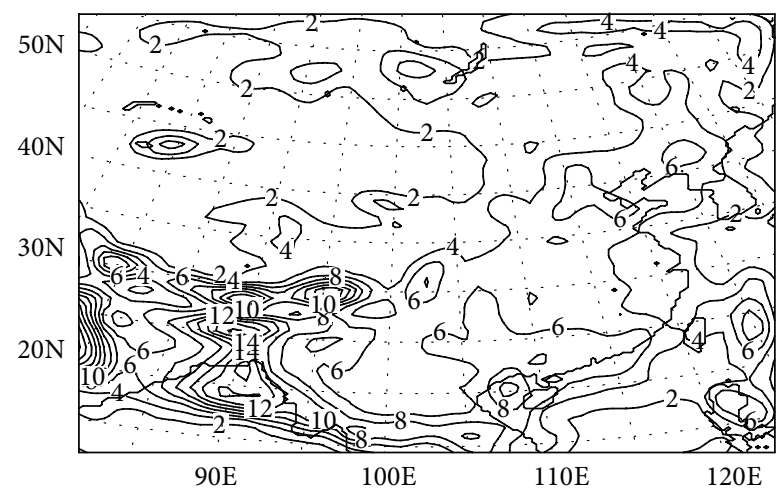

(d) $\mathrm{V} 23 \mathrm{H} 9$

FIGURE 7: Summer-mean precipitation distribution from observations and Scheme V23 for (a) observations, (b) H3, (c) H6, and (d) H9 in $\mathrm{mm} \mathrm{d}^{-1}$.

TABLE 4: Spatial correlation coefficients of precipitation from the L23 and X3 Schemes for the studied areas.

\begin{tabular}{lccccccc}
\hline Test & SC & SWC & EC & NWC & NC & NEC & ALL \\
\hline V23H9 & 0.1409 & -0.1086 & 0.2525 & 0.5853 & 0.5776 & 0.4614 & 0.5136 \\
V23H6 & 0.2861 & -0.0461 & 0.109 & 0.7994 & 0.527 & 0.4634 & 0.5978 \\
V23H3 & 0.1865 & 0.1953 & 0.2872 & 0.8296 & 0.5536 & 0.4351 & 0.6713 \\
V18H3 & 0.3269 & -0.104 & 0.1712 & 0.8604 & 0.5295 & 0.4681 & 0.5968 \\
V14H3 & 0.1867 & 0.2704 & -0.0382 & 0.8688 & 0.5829 & 0.4502 & 0.6407 \\
\hline
\end{tabular}

RMSE and CORR values are the smallest and the largest, respectively, and the $\mathrm{STD}_{M}-\mathrm{STD}_{\mathrm{O}}$ difference is the smallest. Meanwhile, $\mathrm{H} 3$ performs best in East China, and the CORR is generally significantly lower in the south than in the north, with negative CORR values in the south. Overall, the RMSE values in the south are larger than those in the north. For V23, only the medium horizontal resolution (i.e., $60 \mathrm{~km}$ ) produces amounts of precipitation similar to the observations; that is, increasing horizontal resolution does not necessarily lead to better precipitation simulations.

(b) Comparison of the V14 and V18 Schemes with Scheme V23. Figure 6(a) also shows the V18 BIAS values with increasing horizontal resolution; that is, $\mathrm{H} 9, \mathrm{H} 6$, and $\mathrm{H} 3$ BIAS have values of $0.17,0.21$, and $0.05 \mathrm{~mm} \mathrm{~d}^{-1}$, respectively, for the total area. In the subareas, consistently decreasing BIAS values occur at higher horizontal resolutions only in South and North China. By contrast with the other subareas, all V18 BIAS values for East China are negative $(-0.52,-0.51$, and $\left.-1.11 \mathrm{~mm} \mathrm{~d}^{-1}\right)$. Meanwhile, the H9 BIAS value is the smallest in Southwest China. The RMSE value decreases significantly $\left(0.2 \mathrm{~mm} \mathrm{~d}^{-1}\right)$ for the total area in the $\mathrm{H} 6$ experiment. South and North China show the same patterns of change that are observed for the total area. In addition, H9, H6, and H3 present RMSE values of $1.68,1.47$, and $1.94 \mathrm{~mm} \mathrm{~d}^{-1}$ in East China and $1.08,1.07$, and $1.23 \mathrm{~mm} \mathrm{~d}^{-1}$ in Northwest China, respectively. These results indicate that very large changes can occur in the amplitudes of the assessment measures, suggesting that the simulated precipitation is highly sensitive to horizontal resolution in the different subareas.

Considering the BIAS for the total area, Scheme V14 shows that simulated precipitation is not very sensitive to horizontal resolution. However, the subareas show high sensitivity; for example, in North China, the H9-H3 BIAS difference reaches $1.6 \mathrm{~mm} \mathrm{~d}^{-1}$, which is approximately $20 \%$ of the mean summer value for mainland China. In addition, with increasing horizontal resolution, all the RMSE values in North, Northeast, and Southwest China consistently 
decrease. In the total area, the H9 RMSE value is higher than those of $\mathrm{H} 3$ and $\mathrm{H} 6$.

In general, Schemes V14 and V23 appear to perform best at a resolution of $60 \mathrm{~km}$. For Scheme V18, higher horizontal resolutions correspond with closer agreement between the simulated and observed amounts of precipitation. For the total area, the largest BIAS (RMSE) difference for V23 between $\mathrm{H} 3$ and $\mathrm{H} 6$ ( $\mathrm{H} 3$ and $\mathrm{H} 9$ ) is $0.52 \mathrm{~mm} \mathrm{~d}^{-1}$ $\left(0.22 \mathrm{~mm} \mathrm{~d}^{-1}\right)$. Similar results can be observed for Schemes V18 and V14. The influences of horizontal resolution on simulated precipitation are nonlinear at different vertical resolutions. Additionally, the sensitivity of simulated precipitation to horizontal resolution is highest for Scheme V23 and lowest for Scheme V18. The sensitivity in the subareas is stronger than in the total area.

\subsubsection{Vertical Resolution Effects}

(a) Preliminary Results of Resolution H3. The three experiments considering the $\mathrm{H} 3$ resolution result in the simulation of significantly more precipitation than observed precipitation. While V18 presents the smallest BIAS values for the total area (i.e., $0.05 \mathrm{~mm} \mathrm{~d}^{-1}$ ), the $\mathrm{V} 23$ and $\mathrm{V} 14$ values are much larger (i.e., 0.37 and $1.45 \mathrm{~mm} \mathrm{~d}^{-1}$, resp.). In the subareas other than Northwest China, where the V18 BIAS value is greatest, and East China, where the V18 BIAS is the lowest, the results are similar to those for the total area. For the total area, the RMSE values consistently decrease with increasing vertical resolution, and the decrease of V18 (V23) is significant (slight). In the subareas, the V18 RMSE values for South, North, and Northeast China are the smallest, and those for Southwest and East China consistently decrease with increasing vertical resolution.

With higher vertical resolution, the spatial variability of precipitation is closer to the observation of the total area (Figure 5(b)). In the subareas, the $\mathrm{V} 23 \mathrm{STD}_{M}$ values are closest to the observations in East and North China and the V18 values are closest to the observations in South and Northeast China. The V14 $\mathrm{STD}_{M}$ values are far from the observations of all the subareas.

V23 CORR is the largest (Table 4), and V14 has a larger value than V18 in the total area. Notably, CORR shows some results that are inconsistent with the $\mathrm{STD}_{M}$ results (e.g., in Southwest China, V14 $\mathrm{STD}_{M}$ is furthest from the observations and V14 CORR is the highest among the three experiments). In general, for $\mathrm{H} 3$ experiments, V18 shows an obvious improvement in performance relative to V14. However, V23 does not significantly improve the results of V18.

(b) Comparison of the H6 and H9 Resolutions with the H3 Resolution. For the total area, the H6 BIAS values of the V14, V18, and V23 experiments are $1.46,0.21$, and $-0.03 \mathrm{~mm} \mathrm{~d}^{-1}$, respectively. The other subareas, except for Northwest and East China, show that the V14, V18, and V23 absolute BIAS values consistently decrease with increasing vertical resolution, especially V18. For the RMSE, the total area decreases with increasing vertical resolution, with only a slight decrease observed for V23. Except in East China, where the smallest V18 value occurs, all the subareas show results that are similar to those for the total area; for example, the $\mathrm{H} 9$ results are very similar to those of $\mathrm{H} 6$.

Additionally, V18 outperforms V14 in simulated precipitation for the total area. At resolutions of both $\mathrm{H} 6$ and $\mathrm{H} 9$, the V18-V23 precipitation difference is relatively small and the V18 and V23 precipitations are closer to the observations than V14. The largest V18 and V23 decreases in precipitation BIAS (RMSE) are 1.49 and $1.81 \mathrm{~mm} \mathrm{~d}^{-1}\left(0.84\right.$ and $\left.0.94 \mathrm{~mm} \mathrm{~d}^{-1}\right)$, respectively. Therefore, the precipitation sensitivity to vertical resolution is stronger than its sensitivity to horizontal resolution. In the subareas, the simulated precipitation is also sensitive to vertical resolution at different horizontal resolutions, especially for the rainy region of South China.

3.3. Relations between the Effects on SAT and Precipitation. The simulation results also show differences between the effects of resolution on the simulated SATs and precipitation. For Scheme V23, the H6 SAT shows no improvement over the H9 SAT but the H6 precipitation is substantially improved. The H3 SAT improves compared to the H6 SAT, but the improvement in precipitation at $\mathrm{H} 3$ is insignificant. For Scheme V18, the simulated SATs deviate more from the observations as the horizontal resolution increases while the simulated precipitation is closer to the observations. For Scheme V14, the changes in the SAT with horizontal resolution are similar to those of Scheme V18. However, the changes in precipitation with horizontal resolution are similar to those of Scheme V23. Therefore, the SAT and precipitation are well simulated at each resolution and better results are not obtained at certain resolutions. This result clearly shows that different resolutions result in different atmospheric thermodynamic states and suggests that the coordination of the thermodynamic equilibrium of the landatmosphere system is not treated well in the model. Thus, the simulated thermodynamic equilibrium can be modified by changing the resolution and can deviate from the real state. In addition, when comprehensively comparing the effects of resolution on the simulations, the simulations of SAT and precipitation are (or the coordination of the atmospheric thermal field is) the best at a horizontal resolution of $30 \mathrm{~km}$ with 23 vertical layers. Thus, a suitable configuration for horizontal and vertical resolutions exists that can improve the simulation capabilities of the models [42].

Meanwhile, the sensitivity of simulated SAT to resolution is different from that of precipitation. Of the different vertical resolutions, the highest sensitivity of the simulated SAT to horizontal resolution exists in Scheme V18 and the lowest sensitivity exists in Scheme V23. The simulated precipitation results contrast the SAT results. Therefore, at higher vertical resolution, the sensitivity of simulated SAT to horizontal resolution is opposite to that of the simulated precipitation. The SAT and precipitation demonstrate a stronger sensitivity to vertical resolution than horizontal resolution, indicating that the model is sensitive to vertical resolution. The CORRs of simulated precipitation are generally lower than those of SAT, and the change in the CORR of the precipitation with resolution is larger than the changes in the SAT CORR, which 
clearly demonstrates that simulated precipitation is affected more by resolution than SAT.

Considering the effects of resolution on the subareas, the distributions of simulated SATs become similar to the observations with increasing horizontal resolution. However, the spatial variability is not affected much by the vertical resolution. The differences between the distributions of the simulated precipitation and observations are much greater than those for the SAT, and the CORR variations are very large with changes in the horizontal and vertical resolution. Furthermore, the sensitivities of SAT and precipitation to resolution are generally stronger in the subareas than for the total area. The SAT sensitivity to resolution is the largest in Northwest and North China (corresponding to the largest SAT BIAS differences in the different experiments at 1.96 and $0.98^{\circ} \mathrm{C}$, resp.), and the precipitation sensitivity is the largest in Southwest and South China (corresponding to the largest BIAS differences in precipitation of 4.78 and $2.59 \mathrm{~mm} / \mathrm{d}$, resp.). These characteristics are closely related to the dry climates of Northwest and North China and the humid climates of Southwest and South China. Because of the dry climate and low soil heat capacity in the north, the change in resolution can easily cause a change in surface energy flux and land-atmosphere exchange, which would significantly change the SAT. Due to the rainy and humid climate and high soil heat capacity in the south, the change in resolution has a large influence on the grid-scale precipitation and cloud structure. Therefore, different resolutions lead to large precipitation differences in the south and small changes in the simulated SAT.

\section{Summary and Discussion}

In this paper, the regional climate model RegCM3 was used to simulate the 2004 summer surface air temperature and precipitation at three horizontal (i.e., 30, 60, and $90 \mathrm{~km}$ ) and vertical resolutions (i.e., 14, 18, and 23 layers) over the same geographic area (i.e., the area of the model domain excluding the buffer zone) in mainland China. In addition, the effects of resolution on the SAT and precipitation simulations were evaluated using various measures.

The effects of increasing resolution on simulated SAT and precipitation for mainland China are evident. For instance, compared with the increasing summer SAT $\left(0.15^{\circ} \mathrm{C} / 10 \mathrm{a}\right)$ in the last 54 years over China [39], the largest SAT difference among the different experiments for the total area was $0.43^{\circ} \mathrm{C}$ (Figure 3). This result indicates a very strong sensitivity to resolution. In addition, the correlation coefficients show a higher resolution-induced sensitivity to precipitation than that observed for the SAT. At the different vertical resolutions, the effects of the horizontal resolution on the simulated SAT and, particularly, precipitation are apparent with varying features. For example, the 23-layer scheme (i.e., V23) results are not always closer to the observations with increasing horizontal resolution; that is, it performs best at the medium resolution of $60 \mathrm{~km}$ for simulated precipitation and at the highest resolution of $30 \mathrm{~km}$ for simulated SAT. Similarly, at different horizontal resolutions, the effects of increasing vertical resolution on simulated SAT and precipitation are evident, especially for subareas.

The effects of changing the horizontal resolution on the SATs differ from the effect obtained by changing the vertical resolution. Changing the vertical resolution has a stronger influence on the simulated results than changing the horizontal resolution, particularly for precipitation simulations. The distribution of simulated SAT becomes closer to the observations with increasing horizontal resolution; however, this phenomenon is less evident with increasing vertical resolution. This result occurs because higher horizontal resolution (as opposed to higher vertical resolution) increases the more realistic local forcings at the land surface, which greatly affect the SAT [43]. In contrast, the effects of horizontal and vertical resolution changes on precipitation are large. However, the reasons underlying these effects are different and include the following: changes in the topography related to changes in the horizontal resolution, which serves as an important force that affects local precipitation, may modify the simulated precipitation [17]; and the cloud structure change associated with vertical resolution changes may change the simulated precipitation [21].

The effects of changing the resolution on SATs differ from the effects of changing the resolution on precipitation, and the resolution-induced sensitivities show some regional characteristics. For example, the simulated SATs in the north and the simulated precipitation in the south were generally the most sensitive to changes in resolution. The difference between the simulated precipitation and observed precipitation distributions is much larger than that of the simulated SATs and changes greatly with horizontal and vertical resolution. In addition, because of the dry climate and low soil heat capacity in the north, the change in resolution tends to easily cause a change in surface energy flux; therefore, the SAT changes significantly. In contrast, due to the rainy and humid climate and high soil heat capacity in the south, the changes in resolution have a large influence on the grid-scale structure of clouds and therefore precipitation. Consequently, the different resolutions result in little change in simulated temperature but large differences in simulated precipitation in the south.

Relative to the simulated SATs with precipitation, the improvements in the simulated SATs do not correspond with the improvements in precipitation due to the change in resolution, indicating that the different resolutions result in greater modifications in the atmospheric thermodynamic state. For example, the simulations of SAT and precipitation for the total area are best at a resolution of $30 \mathrm{~km}$ with 23 vertical layers. The other configurations result in poorer representations of the thermodynamic state. Therefore, the coordination of the atmospheric thermodynamic state fields can be improved at a suitable horizontal resolution and at a matching suitable vertical resolution.

Theoretically, higher resolutions correspond with smaller truncation errors due to the numerical discretization of the model dynamic framework, resulting in simulation results that are closer to the observations. However, due to a deficiency of RCMs, the relationship between the simulated SAT and the precipitation using RegCM3 could depend 
on the selection of physical parameterization schemes, the simulation area, the buffer zone, or even the initial and lateral conditions that are used $[8,16,43-45]$. In this paper, we preliminarily assessed the effects of resolution on simulated surface climate in a case study. However, further investigations are still needed, for example, to further examine the indepth causes of these effects and to examine the effects of resolution on simulations using different model setups and datasets.

\section{Conflict of Interests}

The authors declare that there is no conflict of interests regarding the publication of this paper.

\section{Acknowledgment}

This work was financially funded by the National Natural Science Foundation of China (Grant nos. 41275012 and 41205073).

\section{References}

[1] F. Giorgi and L. O. Mearns, "Introduction to special section: regional climate modeling revisited," Journal of Geophysical Research, vol. 104, no. 6, pp. 6335-6352, 1999.

[2] Y. G. Mayor and M. D. S. Mesquita, "Numerical simulations of the 1 May 2012 deep convection event over Cuba: sensitivity to cumulus and microphysical schemes in a high-resolution model," Advances in Meteorology, vol. 2015, Article ID 973151, 16 pages, 2015.

[3] H. Paeth, K. Born, R. Podzum, and D. Jacob, "Regional dynamical downscaling over west Africa: model evaluation and comparison of wet and dry years," Meteorologische Zeitschrift, vol. 14, no. 3, pp. 349-367, 2005.

[4] M. Rummukainen, "State-of-the-art with regional climate models," Wiley Interdisciplinary Reviews: Climate Change, vol. 1, no. 1, pp. 82-96, 2010.

[5] F. Feser, B. Rrockel, H. Storch, J. Winterfeldt, and M. Zahn, "Regional climate models add value to global model data: a review and selected examples," Bulletin of the American Meteorological Society, vol. 92, no. 9, pp. 1181-1192, 2011.

[6] M. B. Sylla, A. T. Gaye, and G. S. Jenkins, "On the fine-scale topography regulating changes in atmospheric hydrological cycle and extreme rainfall over West Africa in a regional climate model projections," International Journal of Geophysics, vol. 2012, Article ID 981649, 15 pages, 2012.

[7] M. B. Sylla, J. S. Pal, G. L. Wang, and P. J. Lawrence, "Impact of land cover characterization on regional climate modeling over West Africa," Climate Dynamics, vol. 46, no. 1-2, pp. 637-650, 2016.

[8] F. Giorgi and M. R. Marinucci, "A investigation of the sensitivity of simulated precipitation to model resolution and its implications for climate studies," Monthly Weather Review, vol. 124, no. 1, pp. 148-166, 1996.

[9] X.-M. Zeng, M. Zhao, R.-C. Yu et al., "Application of a 'BigTree' model to regional climate modeling: a sensitivity study," Theoretical and Applied Climatology, vol. 76, no. 3-4, pp. 203218, 2003.
[10] X.-M. Zeng, M. Zhao, B.-K. Su et al., "Effects of the landsurface heterogeneities in temperature and moisture from the 'combined approach' on regional climate: a sensitivity study,' Global and Planetary Change, vol. 37, no. 3-4, pp. 247-263, 2003.

[11] X.-M. Zeng, J. B. Liu, Z. G. Ma, S. Song, C. L. Xi, and H. J. Wang, "Study on the effects of land surface heterogeneities in temperature and moisture on annual scale regional climate simulation," Advances in Atmospheric Sciences, vol. 27, no. 1, pp. 151-163, 2010.

[12] M. B. Sylla, E. Coppola, L. Mariotti et al., "Multiyear simulation of the African climate using a regional climate model (RegCM3) with the high resolution ERA-interim reanalysis," Climate Dynamics, vol. 35, no. 1, pp. 231-247, 2010.

[13] J. Y. Xu, Y. Shi, X. J. Gao, and F. Giorgi, "Projected changes in climate extremes over China in the 21st century from a high resolution regional climate model (RegCM3)," Chinese Science Bulletin, vol. 58, no. 12, pp. 1443-1452, 2013.

[14] C. Torma, E. Coppola, F. Giorgi, J. Bartholy, and R. Pongrácz, "Validation of a high-resolution version of the regional climate model RegCM3 over the Carpathian basin," Journal of Hydrometeorology, vol. 12, no. 1, pp. 84-100, 2011.

[15] X. J. Wang, M. X. Yang, G. N. Wan, X. L. Chen, and G. J. Pang, "Qinghai-Xizang (Tibetan) Plateau climate simulation using the regional climate model RegCM3," Climate Research, vol. 57, no. 3, pp. 173-186, 2013.

[16] L. R. Leung and Y. Qian, "The sensitivity of precipitation and snowpack simulations to model resolution via nesting in regions of complex terrain," Journal of Hydrometeorology, vol. 4, no. 6, pp. 1025-1043, 2003.

[17] X. Gao, Y. Xu, Z. Zhao, J. S. Pal, and F. Giorgi, "On the role of resolution and topography in the simulation of East Asia precipitation," Theoretical and Applied Climatology, vol. 86, no. 1-4, pp. 173-185, 2006.

[18] E. J. Kendon, N. M. Roberts, C. A. Senior, and M. J. Roberts, "Realism of rainfall in a very high-resolution regional climate model," Journal of Climate, vol. 25, no. 17, pp. 5791-5806, 2012.

[19] S. C. Chan, E. J. Kendon, H. J. Fowler, S. Blenkinsop, N. M. Roberts, and C. A. T. Ferro, "The value of high-resolution Met Office regional climate models in the simulation of multihourly precipitation extremes," Journal of Climate, vol. 27, no. 16, pp. 6155-6174, 2014.

[20] P. Hui, J. Tang, S. Wang, J. Wu, X. Niu, and Y. Kang, "Impact of resolution on regional climate modeling in the source region of Yellow River with complex terrain using RegCM3," Theoretical and Applied Climatology, 2015.

[21] P. M. Ruti, D. D. Rocco, and S. Gualdi, "Impact of increased vertical resolution on simulation of tropical climate," Theoretical and Applied Climatology, vol. 85, no. 1-2, pp. 61-80, 2006.

[22] J. P. Tang, M. Zhao, and B. K. Su, "The effects of model resolution on the simulation of regional climate extreme events," Acta Meteorologica Sinica, vol. 21, no. 2, pp. 129-140, 2007.

[23] L. M. Druyan, M. Fulakeza, and P. Lonergan, "The impact of vertical resolution on regional model simulation of the west African summer monsoon," International Journal of Climatology, vol. 28, no. 10, pp. 1293-1314, 2008.

[24] T. L. Delworth, A. Rosati, W. Anderson et al., "Simulated climate and climate change in the GFDL CM2.5 high-resolution coupled climate model," Journal of Climate, vol. 25, no. 8, pp. 2755-2781, 2012.

[25] M. Su and H. Wang, "Decadal co-variability of the summer surface air temperature and soil moisture in China under global 
warming," Chinese Science Bulletin, vol. 52, no. 11, pp. 1559-1565, 2007.

[26] X.-M. Zeng and C.-L. Xi, "Study of the effects of reducing systematic errors on monthly regional climate dynamical forecast," Journal of Tropical Meteorology, vol. 15, no. 1, pp. 102-105, 2009.

[27] J. S. Pal, F. Giorgi, X. Q. Bi et al., "Regional climate modeling for the developing world: the ICTP RegCM3 and RegCNET," Bulletin of the American Meteorological Society, vol. 88, no. 9, pp. 1395-1409, 2007.

[28] M. P. Marcella and E. A. B. Eltahir, "Modeling the summertime climate of Southwest Asia: the role of land surface processes in shaping the climate of semiarid regions," Journal of Climate, vol. 25, no. 2, pp. 704-719, 2012.

[29] R. L. Gianotti, D. F. Zhang, and E. A. B. Eltahir, "Assessment of the regional climate model version 3 over the maritime continent using different cumulus parameterization and land surface schemes," Journal of Climate, vol. 25, no. 2, pp. 638-656, 2012.

[30] G. A. Grell, J. Dudhia, and D. R. Stauffer, "A description of the fifth-generation Penn State/NCAR mesoscale model (MM5)," NCAR Technical Note NCAR/TN-398+STR, 1994.

[31] A. A. M. Holtslag, E. I. F. De Bruijn, and H.-L. Pan, "A high resolution air mass transformation model for short-range weather forecasting," Monthly Weather Review, vol. 118, no. 8, pp. 1561-1575, 1990.

[32] J. S. Pal, E. E. Small, and E. A. B. Eltahir, "Simulation of regionalscale water and energy budgets: representation of subgrid cloud and precipitation processes within RegCM," Journal of Geophysical Research: Atmospheres, vol. 105, no. 24, pp. 2957929594, 2000.

[33] G. A. Grell, "Prognostic evaluation of assumptions used by cumulus parameterizations," Monthly Weather Review, vol. 121, no. 3, pp. 764-787, 1993.

[34] J. T. Kiehl, J. J. Hack, G. B. Bonan, B. A. Boville, and B. P. Briegleb, "Description of the NCAR community climate model (CCM3)," NCAR Technical Note NCAR/TN-420+STR, 1996.

[35] X.-M. Zeng, B. Wang, Y. Zhang et al., "Effects of land surface schemes on WRF-simulated geopotential heights over China in summer 2003," Journal of Hydrometeorology, 2016.

[36] E. Kalnay, M. Kanamitsu, R. Kistler et al., "The NCEP/NCAR 40-year reanalysis project," Bulletin of the American Meteorological Society, vol. 77, no. 3, pp. 437-471, 1996.

[37] C. J. Willmott, K. Matsuura, and D. L. Legates, "Global air temperature and precipitation: regridded monthly and annual climatologies (version 2.01)," 2013, http://climate.geog.udel.edu/.

[38] L. S. Lian and J. Shu, "Numerical simulation of summer climate over center and east China using a regional climate model," Journal of Tropical Meteorology, vol. 23, no. 2, pp. 162-170, 2007 (Chinese).

[39] G. Y. Ren, M. Z. Xu, Z. Y. Chu et al., "Changes of surface air temperature in China during 1951-2004," Climatic and Environmental Research, vol. 10, no. 4, pp. 717-727, 2005 (Chinese).

[40] D. F. Zhang, X. J. Gao, Z. C. Zhao, J. S. Ral, and F. Giogri, "Simulation of climate in China by RegCM3 model," Advances in Climate Change Research, vol. 1, no. 3, pp. 119-121, 2005 (Chinese).

[41] F. Giorgi, G. T. Bates, and S. J. Nieman, "Simulation of the arid climate of the southern Great Basin using a regional climate model," Bulletin of the American Meteorological Society, vol. 73, no. 11, pp. 1807-1822, 1992.
[42] D. X. Liao and Y. Q. Zhu, "Further studies of consistent horizontal and vertical resolution," Acta Meteorologica Sinica, vol. 52, no. 2, pp. 129-137, 1995 (Chinese).

[43] X.-M. Zeng, N. Wang, Y. Wang et al., "WRF-simulated sensitivity to land surface schemes in short and medium ranges for a high-temperature event in East China: a comparative study," Journal of Advances in Modeling Earth Systems, vol. 7, no. 3, pp. 1305-1325, 2015.

[44] L. F. Li, W. H. Li, and J. M. Jin, "Improvements in WRF simulation skills of southeastern United States summer rainfall: physical parameterization and horizontal resolution," Climate Dynamics, vol. 43, pp. 2077-2091, 2014.

[45] J. T. Bacmeister, M. F. Wehner, R. B. Neale et al., "Exploratory high-resolution climate simulations using the Community Atmosphere Model (CAM)," Journal of Climate, vol. 27, no. 9, pp. 3073-3099, 2014. 

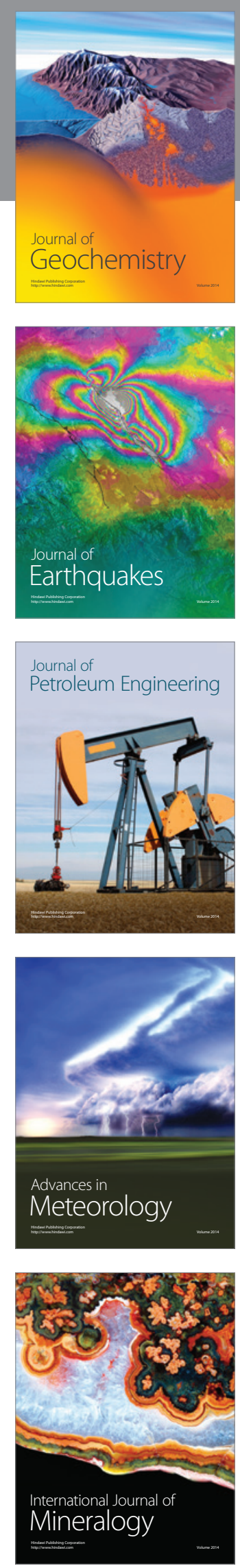
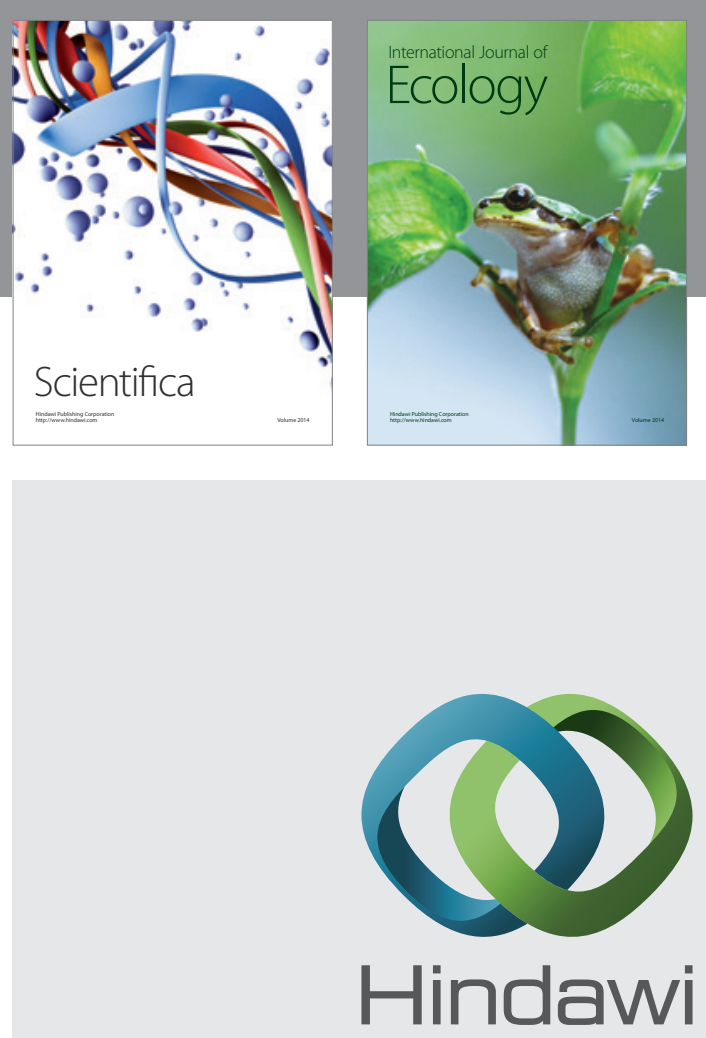

Submit your manuscripts at

http://www.hindawi.com
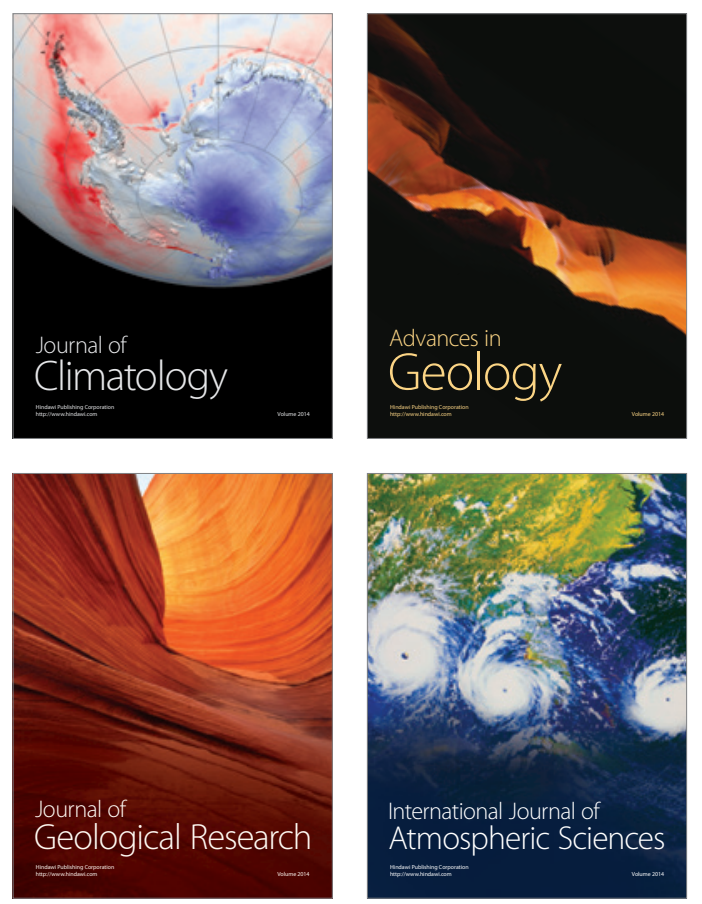

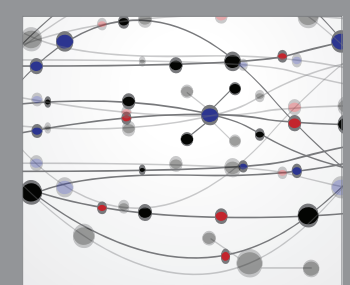

The Scientific

\section{World Journal}
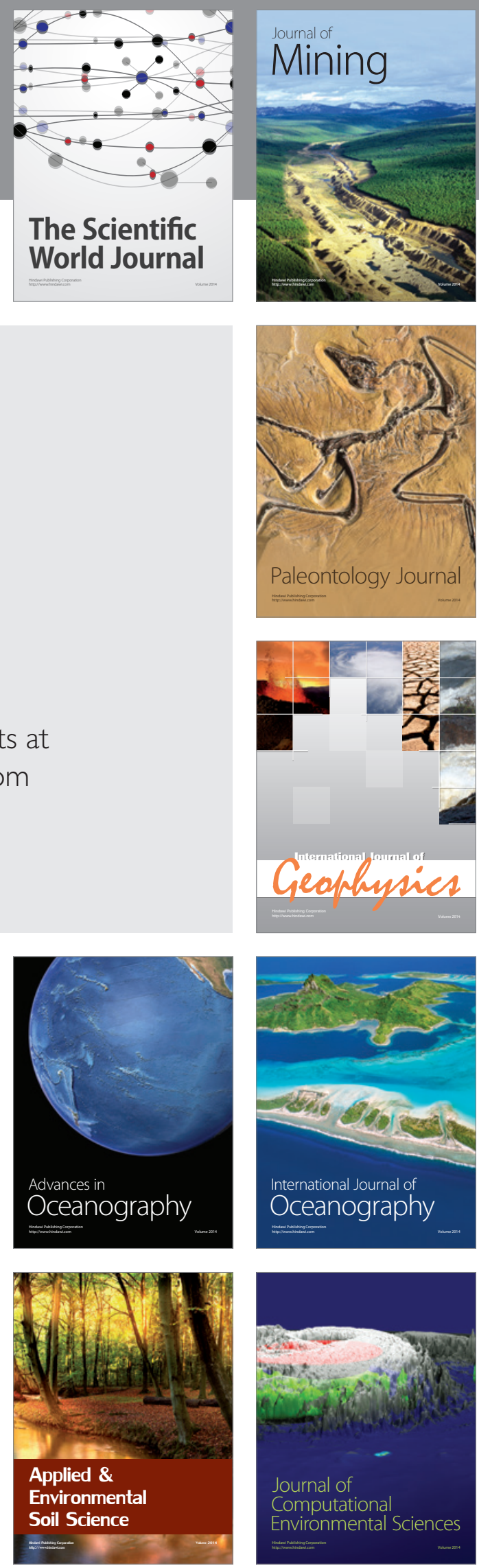